\title{
Targeted RNA Sequencing of Formalin-Fixed, Paraffin-Embedded Temporal Arteries From Giant Cell Arteritis Cases Reveals Viral Signatures
}

Andrew N. Bubak, PhD, Teresa Mescher, BS, Michael Mariani, PhD, Seth E. Frietze, PhD, James E. Hassell, Jr., PhD, Christy S. Niemeyer, PhD, Christina N. Como, BA, Anna M. Burnet, BS, Prem S. Subramanian, MD, PhD, Randall J. Cohrs, PhD, Ravi Mahalingam, PhD, and Maria A. Nagel, MD

Neurol Neuroimmunol Neuroinflamm 2021;8:e1078. doi:10.1212/NXI.0000000000001078

\section{Abstract}

\section{Background and Objectives}

Varicella zoster virus (VZV) antigen has been detected in temporal arteries (TAs) of individuals with giant cell arteritis (GCA), the most common systemic vasculitis in older adults. Thus, we explored the contribution of VZV to GCA pathogenesis.

\section{Methods}

Formalin-fixed, paraffin-embedded TA sections from biopsy-positive GCA participants with VZV antigen (GCA/VZV-positive; $\mathrm{n}=20)$ and without (GCA/VZV-negative, $\mathrm{n}=20$ ) and from normal participants with VZV antigen (control/VZV-positive, $\mathrm{n}=11$ ) and without (control/ VZV-negative, $n=20$ ) were analyzed by targeted RNA sequencing of the whole human transcriptome (BioSpyder TempO-Seq). Ingenuity pathway analysis and R-computational program were used to identify differentially expressed genes and pathways between groups.

\section{Results}

Compared with control/VZV-negative TAs, GCA/VZV-negative and GCA/VZV-positive TAs were significantly enriched for human transcripts specific for pathways involved in viral infections, including viral entry, nuclear factor kappa B activation by viruses, and other pathogenrelated immune activation pathways. Similarly, human gene sets supporting viral infection were found in control/VZV-positive TAs that showed no morphological signs of inflammation, suggesting that the enriched pathways were not nonspecific signatures of infiltrating immune cells. All GCA TAs and control/VZV-positive TAs showed enrichment of transcripts involved in vascular remodeling, including smooth muscle cell migration.

\section{Discussion}

The detection of viral and immune activation pathways in GCA TAs supports a role for virus infection in GCA pathogenesis. In addition, the detection of viral pathways in control/VZVpositive TAs, along with vascular remodeling pathways, suggests that these samples may represent early infection with progression to clinical disease, depending on host and other environmental factors.

\author{
Correspondence \\ Dr. Nagel \\ maria.nagel@ucdenver.edu
}




\section{Glossary}

COMIRB = Colorado Multiple Institutional Review Board; FFPE $=$ formalin-fixed, paraffin-embedded; GCA $=$ giant cell arteritis; gE = glycoprotein E; GSEA = Gene Set Enrichment Analysis; HSV = herpes simplex virus; IFN = interferon; IHC = immunohistochemistry; IL = interleukin; TA = temporal artery; TLR = toll-like receptor; $\mathbf{T N F}=$ tumor necrosis factor; $\mathbf{V Z V}=$ varicella zoster virus.

Giant cell arteritis (GCA), the most common systemic vasculitis in the elderly, has been proposed to be a form of extracranial varicella zoster virus (VZV) vasculopathy ${ }^{1}$ because both VZV vasculopathy and GCA (1) affect the elderly; (2) can present with vision loss, headaches, and stroke ${ }^{2-5}$; and (3) produce transmural arterial inflammation with giant/ epithelioid cells and medial damage in temporal, cerebral, and/or other systemic arteries. ${ }^{6-9}$ Importantly, $70 \%$ of biopsypositive GCA temporal arteries (TAs) contain VZV antigen compared with $18 \%$ of controls. ${ }^{10}$ Follow-up studies by other groups yielded variable frequencies of VZV antigen, ${ }^{1-15}$ raising the question of whether virus contributes to GCA pathogenesis. Previously, gene expression analyses of GCA TAs that would identify viral signatures were challenging because large numbers of fresh TAs were difficult to obtain and formalin-fixed, paraffin-embedded (FFPE) TAs had degraded RNA. Recently, a targeted RNA sequencing assay for the whole human transcriptome (TempO-Seq; BioSpyder Technologies, Carlsbad, CA) was developed that (1) can directly amplify fragmented human RNA in FFPE slides without a reverse transcription step and (2) has results equivalent to the analysis of fresh or frozen tissue. ${ }^{16}$ This technology advancement has allowed us to determine the transcriptional profiles of specific VZV antigen-containing regions within single, FFPE GCA TA slides and compare with control TAs to elucidate the role of virus infection in GCA pathogenesis.

\section{Methods}

\section{Standard Protocol Approvals, Registrations, and Patient Consents}

Archived patient samples were deidentified and deemed exempt not human research as defined by the Colorado Multiple Institutional Review Board (COMIRB) policies and current regulations and in accordance with Office for Human Research Protection and Food and Drug Administration guidelines (Protocol no. 13-2550; COMIRB, 303-724-1055).

\section{Patient Samples}

Forty FFPE TA biopsies from patients with GCA older than 50 years were used; all GCA TAs were biopsy-positive with transmural inflammation, giant/epithelioid cells, and medial damage. Controls were 31 normal TAs (without zoster, diabetes, cancer, substance abuse, or immunosuppression) removed postmortem from participants $>50$ years of age at the University of Colorado Hospital, Arapahoe County Coroner's Office, and Denver Office of the Medical Examiner. These TAs were previously obtained and analyzed by immunohistochemistry (IHC) and quantitative PCR for the presence of VZV antigen and DNA, respectively. ${ }^{1,10}$ Previously published data showed that of the 40 GCA TAs, 16 contained VZV antigen with corresponding DNA from the scraped VZV antigen-positive fields and 4 contained VZV antigen only (20 GCA/VZV-positive TAs); 20 did not contain VZV antigen (20 GCA/VZV-negative TAs). Of 31 control TAs, 3 contained VZV antigen with corresponding DNA and 8 contained VZV antigen only ( 11 control/VZV positive TAs); 20 did not contain VZV antigen (20 control/ VZV-negative TAs). ${ }^{1}$ Patient sample information is in $\mathrm{eTa}$ ble 1 (links.lww.com/NXI/A574).

\section{FFPE Tissue Sequencing and Analysis}

For whole human transcriptome analysis, FFPE slides from TAs were assayed using TempO-Seq-targeted RNA sequencing plates, reagents, protocols, and software (BioSpyder Technologies). TempO-Seq exclusively detects human transcripts; viral transcripts will not be detected. For slides with VZV antigen, the same antigen-containing regions on unstained adjacent slides were measured, scraped $\left(10 \mathrm{~mm}^{2}\right.$ pooled regions of interest per participant), and placed into PCR tubes containing $1 \mathrm{X}$ lysis buffer. For slides without VZV antigen, equivalent areas of arteries from unstained slides were analyzed. Using a thermocycler, samples were lysed, coded adjacent primer pairs for each specific human transcript were annealed to sample RNA, and primer pairs for each transcript ligated and then amplified per manufacturer's instructions. ${ }^{16}$ If a transcript is present, the adjacent 25-nucleotide primer pairs anneal to their specific target, ligate together, and produce a 50-nucleotide transcript-specific, coded amplicon. Amplified PCR products were pooled into a single library, concentrated using a PCR cleanup kit (Macherey-Nagel, Düren, Germany), and run on the Illumina NextSeq 500 sequencing platform (Illumina Inc., San Diego, CA). Mapped reads were generated by TempO-SeqR for the alignment of demultiplexed FASTQ files from the sequencer to the ligated detector oligomer gene sequences using Bowtie, ${ }^{17}$ allowing for up to 2 mismatches in the 50-nucleotide target sequence. ${ }^{16}$ Counts were assessed using SARTools. ${ }^{18}$ Within this $\mathrm{R}$ package, edgeR is used for normalization and quality control of count data. ${ }^{19}$ Raw/normalized counts and multidimensional scaling between the 4 groups are shown in eFigure 1 (links.lww.com/NXI/A571). Differential expression between groups was assessed by the TempO-SeqR software, which used the DESeq2 method for differential analysis of count data. ${ }^{20} \mathrm{~A}$ significantly differentially expressed gene is defined as having an adjusted $p$ value $<0.05$ with no fold-change threshold. 
Pathway enrichment analysis was performed using gene sets and pathways defined in the Ingenuity Pathway Analysis software (Qiagen, Germantown, MD) and the ClusterProfiler package $^{21,22}$ in $\mathrm{R}$ with default parameters. ${ }^{23}$ ClusterProfiler supports enrichment analysis of Gene Ontology and Kyoto Encyclopedia of Genes and Genomes databases with Gene Set Enrichment Analysis (GSEA) to identify biological themes of a collection of genes. These functional enrichment analyses use computational approaches to identify groups of experimentally observed human genes that are overrepresented or depleted in a curated disease or biological function-specific gene set. Additional figures were created using Prism 9 (GraphPad Software, San Diego, CA).

\section{IHC Analysis}

To confirm sequencing results, 5- $\mu \mathrm{m}$ FFPE sections from 10 GCA/VZV-positive TAs and 10 control/VZV-negative TAs were immunostained for interleukin (IL)-8 and neutrophils (CD15). After deparaffinization, antigens were retrieved in citrate buffer at $95^{\circ} \mathrm{C}$ for 10 minutes. Slides were immunostained as described ${ }^{1}$ using primary antibodies against IL-8 (mouse anti-IL-8; 1:500 dilution; Abcam, Cambridge, MA; catalog \#ab18672) and CD15 (mouse anti-CD15; 1:50 dilution; Abcam; catalog \#ab188610). IL-8 and CD15 positive control was normal human tonsil. Slides were imaged by light microscopy.

\section{Data Availability}

The datasets generated and/or analyzed are available on the NCBI Gene Expression Omnibus database (GSE174694).

\section{Results}

\section{Gene Expression Analysis of Control/VZV- Negative and Control/VZV-Positive TAs}

The 31 control TAs were previously immunostained for VZV glycoprotein $\mathrm{E}(\mathrm{gE})$ (50 sections from each artery); 20 were negative for VZV gE and 11 were positive for VZV gE (representative staining, Figure 1A). Compared with control/ VZV-negative TAs, control/VZV-positive TAs had 78 differentially expressed genes ( $p$ adj $<0.05 ; 41$ upregulated [red] and 37 downregulated [blue]; Figure 1B; gene list in eTable 2, links.lww.com/NXI/A575). Control/VZV-positive TAs were enriched for pathways involved in viral infections, fibroblast proliferation, and vascular cell migration/spreading (Figure 1C; full gene list in eTable 3, links.lww.com/NXI/ A576). For example, the viral infection pathway genes IFITM2 and IFITM3 encoding interferon (IFN)-inducible transmembrane proteins that function in viral resistance ${ }^{24}$ were upregulated in control/VZV-positive TAs. Multiple activated (HRAS and ESR1; red) and repressed (MYCN; blue) upstream regulators were identified in control/VZV-positive

Figure 1 Gene Expression Analysis of Control TAs Without and With VZV Antigen

A

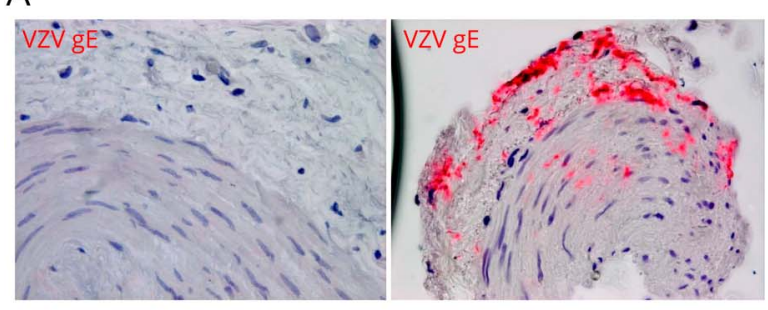

C

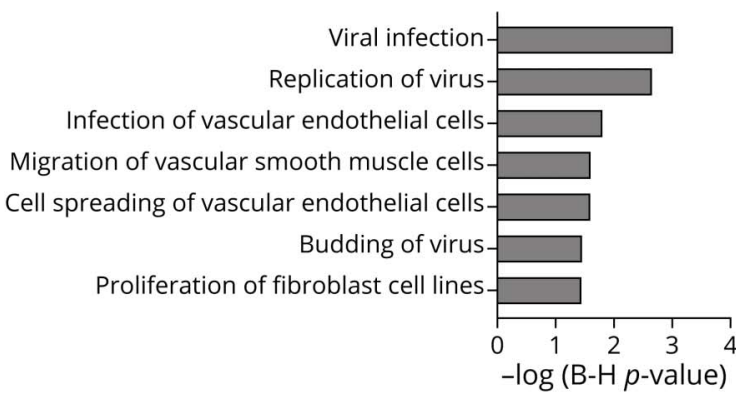

B

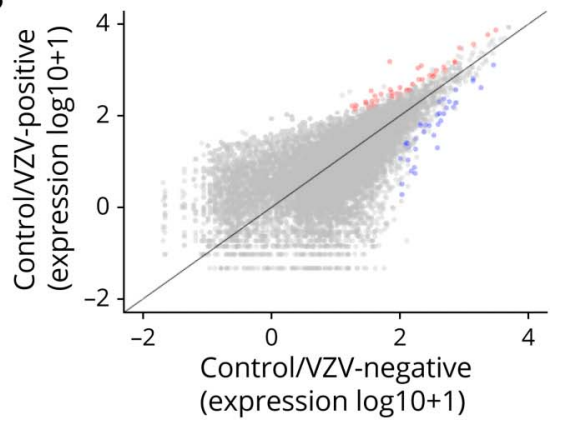

D

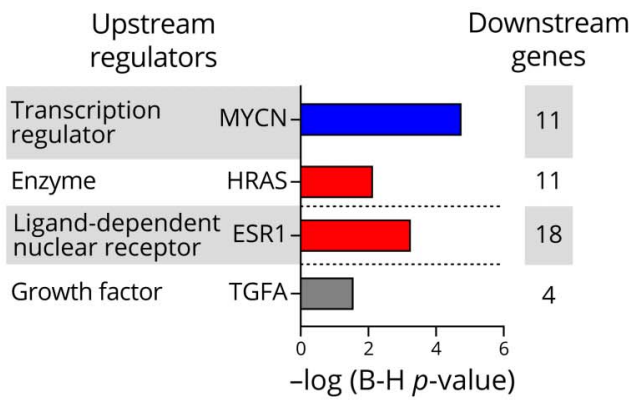

(A) TAs were analyzed by immunohistochemistry for VZV antigen; representative arteries show the absence and presence of VZV antigen staining (left and right panels, control/VZV-negative and control/VZV-positive, respectively; magnification $\times 600$ ). Targeted RNA sequencing of the whole human transcriptome and gene expression/pathway analysis of control/VZV-positive compared with control/VZV-negative TAs revealed distinct differences. (B) Scatterplot analysis showed significantly upregulated (red) and downregulated (blue) genes. (C) Compared with control/VZV-negative TAs, control/VZV-positive TAs were enriched for pathways involved in viral infection, cell proliferation, and migration. (D) Upstream regulators (transcription regulators, enzymes, ligand-dependent nuclear receptors, and growth factors) and corresponding number of downstream genes were identified in control/VZV-positive compared with control/VZVnegative TAs (red signifies activated, blue signifies repressed, and gray signifies unknown activation pattern of transcripts). $g E=$ glycoprotein $\mathrm{E}$; TA = temporal artery; VZV = varicella zoster virus. 
TAs (Figure 1D). Overall, these results support a transcriptional profile indicative of an active viral infection in control TAs that contained VZV antigen, corroborating IHC detection of VZV gE in these samples.

\section{Gene Expression Analysis of Control/VZV- Negative and GCA/VZV-Positive TAs}

The 40 GCA TAs were previously analyzed for VZV gE antigen and VZV DNA. All GCA TAs displayed classic biopsypositive GCA features: transmural inflammation, medial damage, and multinucleated giant/epithelioid cells (Figure 2A, left panel). Twenty TAs contained VZV gE (GCA/VZV-positive) of which 16 were confirmed to have amplifiable VZV DNA (Figure 2A, representative GCA TA with VZV gE). The remaining $20 \mathrm{TAs}$ did not contain VZV gE (GCA/VZV-negative). Compared with control/VZVnegative TAs, GCA/VZV-positive TAs had 1,353 differentially expressed genes ( $p$ adj $<0.05 ; 1,095$ upregulated [red] and 258 downregulated [blue]; Figure 2B; gene list in eTable 2, links.lww.com/NXI/A575). Top canonical pathways revealed known pathways associated with GCA, ${ }^{25,26}$ including Th1 and Th2 pathways, CD28 signaling in T-helper cells, T-cell exhaustion, IFN signaling, neuroinflammation signaling, and toll-like receptor (TLR) signaling (Figure 2C). In addition, defined canonical pathways that included virus entry through endocytic pathways were enriched in GCA/VZVpositive samples (Figure 2C; red signifies activation, blue signifies inhibition, and gray signifies unavailable activation pattern). This involved a number of genes encoding endocytic components such as clathrin (CLTA) and the actin-related proteins (ARPC2, ACTR3, and ACTR1B). Multiple cytokines were identified as upstream regulators for GCA/VZV-positive TAs compared with control/VZV-negative TAs, including IFN $\gamma$, tumor necrosis factor (TNF), IL-1B, IL-2, IL-15, IL-6, and IL-8 (Figure 2D). In addition, several growth factors (TGFB1 and KITLG), transcription regulators (STAT1, STAT3, IRF7, and SIRT1), TLRs (TLR3, TLR4, and TLR9), and the transferrin receptor (TFRC) were also linked as key upstream regulators. The major histocompatibility complex class II gene, human leukocyte antigen (HLA)-DRB1, expression was higher in GCA/VZV-positive TAs compared with control/VZV-negative TAs $(3.51 \pm 1.21$; $\log 2$ FoldChange \pm standard error, $p$-adj $<0.05)$, which has been previously reported as a GCA risk factor (reviewed by Carmona et $\mathrm{al}^{27}$ ).

\section{Viral Infection and Immune Responses Are Enriched in GCA/VZV-Positive TAs Compared With Control/VZV-Negative TAs}

Further investigations into GCA/VZV-positive TAs revealed enrichment of vascular and arterial disease gene sets associated with activated viral infection and neutrophil degranulation pathways (Figure 3A; full gene list in eTable 3, links.lww.com/NXI/A576). Differentially expressed human transcripts associated with advanced-stage peripheral arterial disease, degranulation of neutrophils, and viral infection showed similar positive expression directionality (Figure 3B) and gene set overlap (Figure 3C). Running enrichment scores in the GSEA showed an increase in neutrophil degranulation, virus diseases, and vasculitis in GCA/VZV-positive TAs compared with control/VZV-negative TAs and a decrease in muscle structure development (Figure 3D). The presence of IL-8 (a chemoattractant of neutrophils) and CD15 (marker of neutrophils) was confirmed in all $10 \mathrm{GCA} / \mathrm{VZV}$-positive TAs examined (representative TA, second row, eFigure 2, links.lww. com/NXI/A572). No control/VZV-negative TAs were positive for IL-8 (third row, eFigure 2), and only 1 was positive for CD15 in the 10 samples examined. Human tonsils were a positive control for IL-8 and CD15 (first row, eFigure 2). No staining was observed for IL-8 or CD15 when primary antibodies were substituted with $\mathrm{mIgG1}$ isotype control antibody (second and fourth columns; eFigure 2).

\section{Viral Infection and Immune Responses Are Enriched in GCA/VZV-Negative TAs Compared With Control/VZV-Negative TAs}

A subset of TAs from patients with GCA were negative for VZV antigen (GCA/VZV-negative). We investigated whether this group is reflective of a nonspecific inflammatory response or potentially represents TAs where detection of VZV antigen (or another virus) was missed because of skip lesions. ${ }^{1}$ Compared with control/VZV-negative TAs, GCA/VZVnegative TAs had 1,784 differentially expressed genes (padj $<0.05 ; 1,378$ upregulated [red] and 406 downregulated [blue]; Figure 4A; gene list in eTable 2, links.lww.com/NXI/ A575). Top canonical pathways of GCA/VZV-negative and GCA/VZV-positive TAs were similar; compared with control/VZV-negative TAs, GCA/VZV-negative TAs were enriched for Th1 and Th2 pathways, CD28 signaling in T-helper cells, T-cell exhaustion, IFN signaling, and neuroinflammation signaling (Figure 4B; red signifies activation, blue signifies inhibition, and gray signifies unavailable activation pattern). As was seen in GCA/VZV-positive TAs, GCA/VZV-negative TAs also showed enrichment for pathways associated with viral infections including nuclear factor kappa $B$ activation by viruses and virus entry through endocytic pathways (Figure 4B). Furthermore, analysis of enrichment scores through GSEA revealed activated gene sets reflective of viral infections, including defense response to virus and TLR signaling (Figure 4C). This included upregulated expression of genes JAK1, JAK2, STAT1, and STAT2 of the JAK-STAT signaling pathway in GCA/VZVnegative TAs. Upstream regulators of differentially expressed genes in GCA/VZV-negative TAs were largely similar to GCA/VZV-positive TAs such as cytokines (IFN $\gamma$, TNF, IL-1B, IL-2, IL-15, IL-6, and IL-8), the growth factor TGFB1, transcription regulators (STAT1, STAT3, and IRF7), TLRs (TLR3 and TLR4), and the iron transporter TFRC (eFigure 3, links.lww.com/NXI/A573; red signifies activation and blue signifies repression). 
A
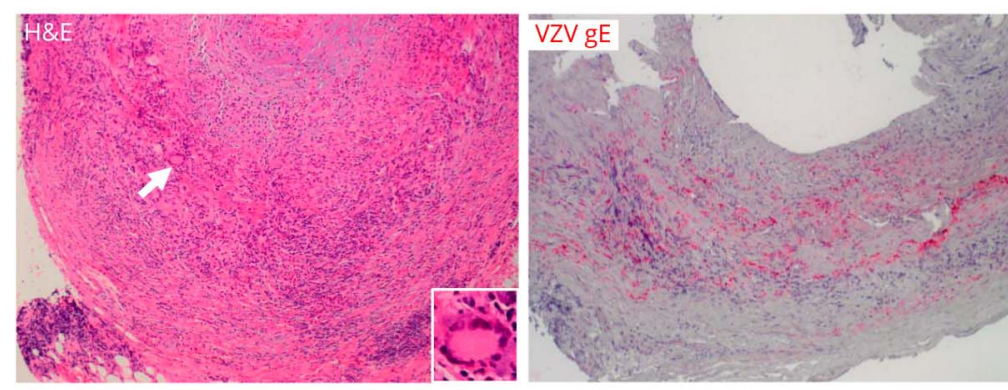

C
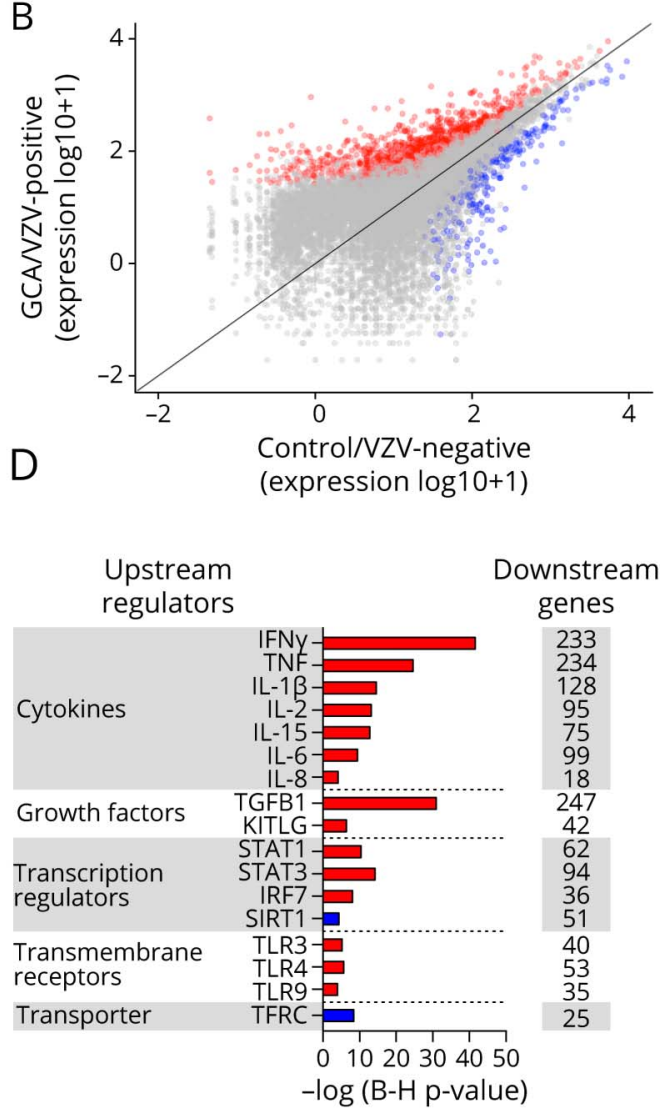

(A) GCA/VZV-positive TAs that contained VZV antigen by immunohistochemistry were previously stained with hematoxylin and eosin to confirm GCA pathology. ${ }^{1}$ A representative GCA/VZV-positive TA is shown (left panel; magnification $\times 100$ ) with transmural inflammation, medial damage, and multinucleated giant cells (left panel, arrow and inset), as well another GCA/VZV-positive TA containing VZV antigen (right panel, red). Targeted RNA sequencing of the whole human transcriptome and gene expression/pathway analysis of GCA/VZV-positive TAs compared with control/VZV-negative TAs revealed distinct differences. (B) Scatterplot analysis showed significantly upregulated (red) and downregulated (blue) genes in GCA/VZV-positive TAs compared with control/ VZV-negative TAs. (C) Top canonical pathways identified in GCA/VZV-positive vs control/VZV-negative TAs indicated that GCA/VZV-positive TAs seem to activate immune responses and viral pathways. (D) Key upstream regulators (cytokines, growth factors, transcription regulators, transmembrane receptors, and transporters) and corresponding number of downstream genes were identified in GCA/VZV-positive TAs compared with control/VZV-negative TAs (red signifies activated and blue signifies repressed). GCA = giant cell arteritis; gE = glycoprotein E; IFN = interferon; IL = interleukin; TA = temporal artery; TNF = tumor necrosis factor; VZV, varicella zoster virus.

\section{Predicted Upstream Regulators and Pathways Generated From Unique Differentially Expressed Genes in GCA/VZV-Negative and GCA/VZV-Positive TAs Compared With Control/ VZV-Negative TAs}

Although GCA/VZV-positive and GCA/VZV-negative TAs displayed similar pathways and upstream regulators compared with control/VZV-negative TAs, we investigated the extent of differentially expressed genes that were shared or unique between GCA TAs without or with VZV antigen compared with control/VZV-negative TAs. GCA/VZVnegative and GCA/VZV-positive TAs shared 1,111 genes that were differentially expressed compared with control/ VZV-negative TAs (Figure 5A, middle). Conversely, GCA/
VZV-negative vs control/VZV-negative TAs had 673 unique differentially expressed genes that were not found in GCA/ VZV-positive vs control/VZV-negative TAs (Figure 5A, left). A total of 249 genes were unique to GCA/VZVpositive vs control/VZV-negative TAs that were not found in GCA/VZV-negative vs control/VZV-negative TAs (Figure 5A, right). Upstream regulators for GCA/VZVnegative unique genes included IL-4, EIF4E, and TGFB1 (Figure 5B, left, red signifies activated); upstream regulators for GCA/VZV-positive unique genes included IFN $\gamma$, TNF, and myocardin-related transcription factor A (MRTFA) (Figure 5B, right, red signifies activated and blue signifies inhibited). Effects of decreased MRTFA in our samples remain to be determined because increased MRTFA has been 

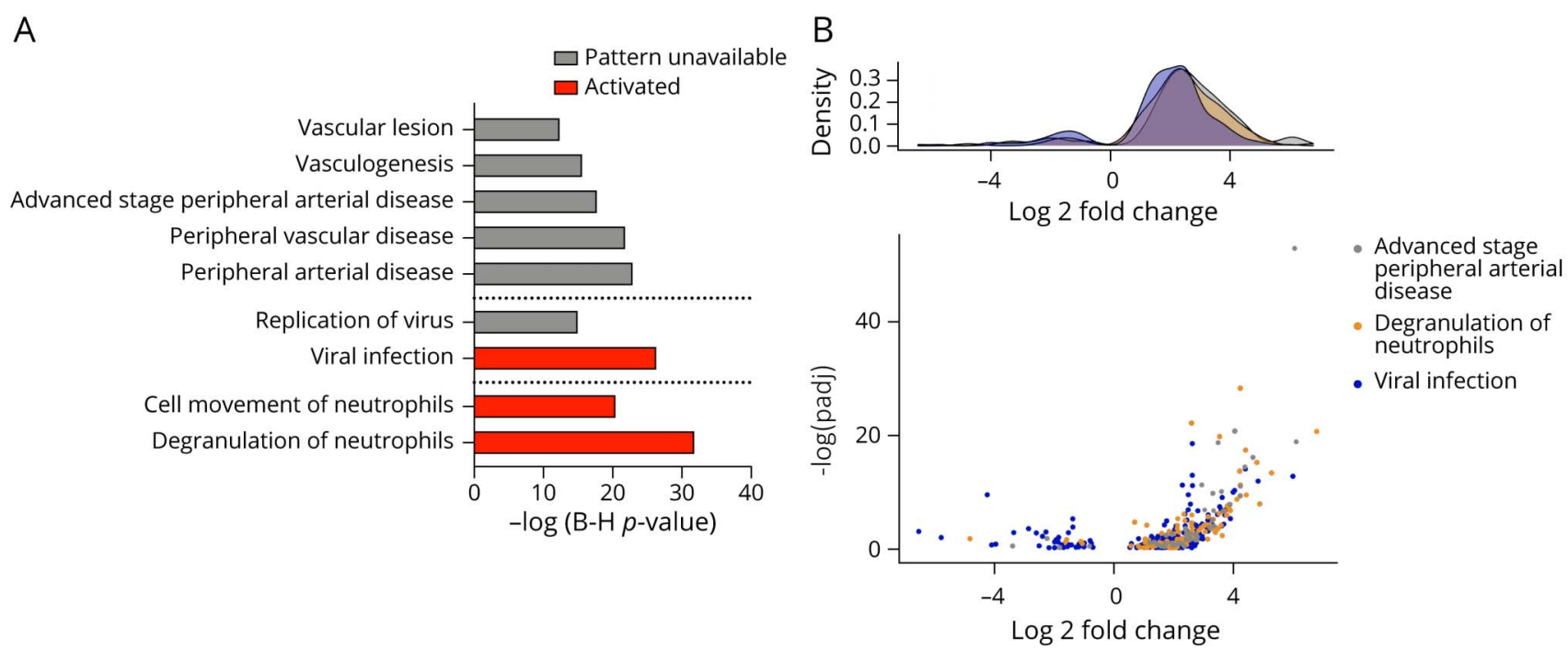

C

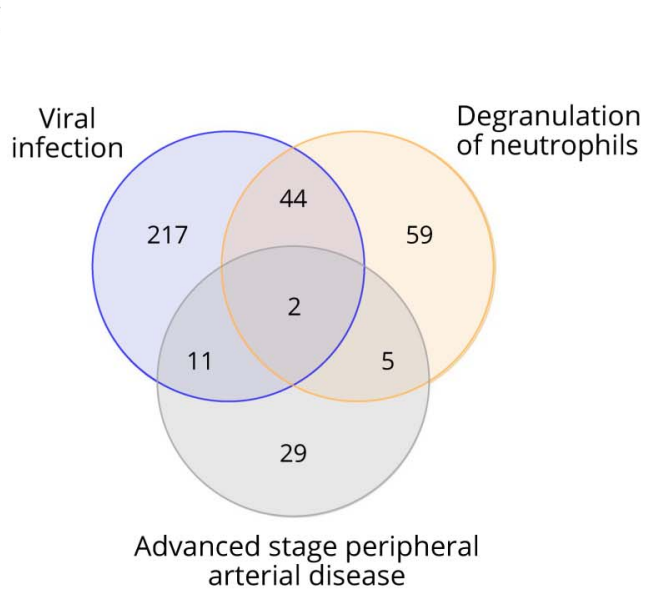

D.a Neutrophil degranulation

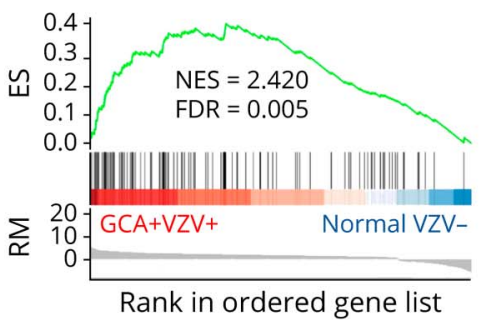

D.b virus diseases

D.c Vasculitis
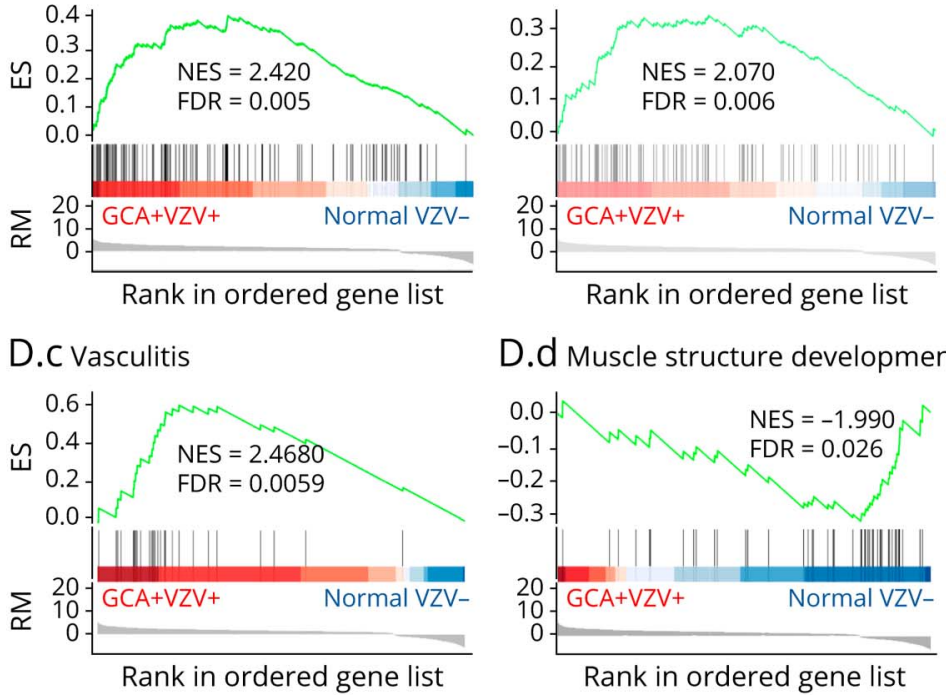

D.d Muscle structure development

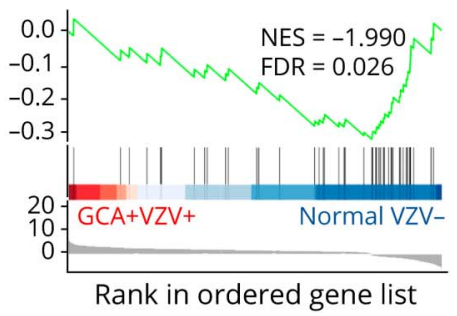

(A) Human gene sets involved in vascular and arterial disease, viral infections, and neutrophil movement and degranulation were significantly enriched in GCA/ VZV-positive TAs compared with control/VZV-negative TAs (red signifies activated and gray signifies unknown activity pattern). (B) Volcano and density plot showing distribution and direction of differentially expressed genes involved in advanced stage peripheral arterial disease (gray), degranulation of neutrophils (gold), and viral infection (blue) measured in GCA/VZV-positive TAs compared with control/VZV-negative TAs. (C) Venn diagram showing independent and shared differentially expressed genes between viral infection (blue), degranulation of neutrophils (gold), and advanced stage peripheral arterial disease (gray) gene sets measured in GCANZV-positive TAs. (D) In GCANVZV-positive TAs, gene sets were positively enriched for neutrophil degranulation, virus diseases, and vasculitis but negatively enriched for muscle structure development. The $y$-axis represents ES and RM (significant genes ranked by fold-change); $x$-axis are genes (vertical black lines) represented in the ranked order gene sets. The green line connects points of ES and genes (NES). ES = enrichment score; FDR = false discovery rate; $\mathrm{GCA}$ = giant cell arteritis; NES = normalized enrichment score; RM = ranked metric; TA = temporal artery; VZV, varicella zoster virus.

associated with cell migration and pathologic vascular remodeling in mice. ${ }^{28}$ Enrichment analysis of GCA/VZV-negative unique differentially expressed genes (DEGs) revealed pathways and gene sets associated with cell movement of leukocytes, differentiation of mononuclear leukocytes, replication of Herpesviridae, and viral infection (Figure 5C, left; full gene list in eTable 3, links.lww.com/NXI/A576). Associated pathways and gene sets for GCA/VZV-positive unique DEGs included inflammatory response, peripheral vascular disease, systemic autoimmune syndrome, and viral infection (Figure 5C, right; full gene list in eTable 2, links.lww.com/NXI/A575). Examples of inflammatory genes upregulated included JAK1, TNFRSF10A, NFATC1, and NFKB1.

\section{Discussion}

VZV vasculopathy was initially described as virus infection of cerebral arteries leading to ischemic or hemorrhage stroke, aneurysm, or other cerebrovascular abnormalities that could occur with or without associated rash. Subsequently, VZV vasculopathy expanded to include the extracranial circulation 


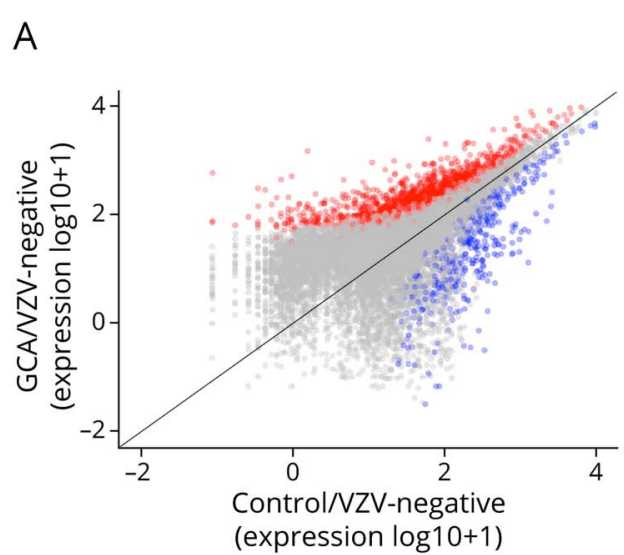

C.a Defense reponse to virus

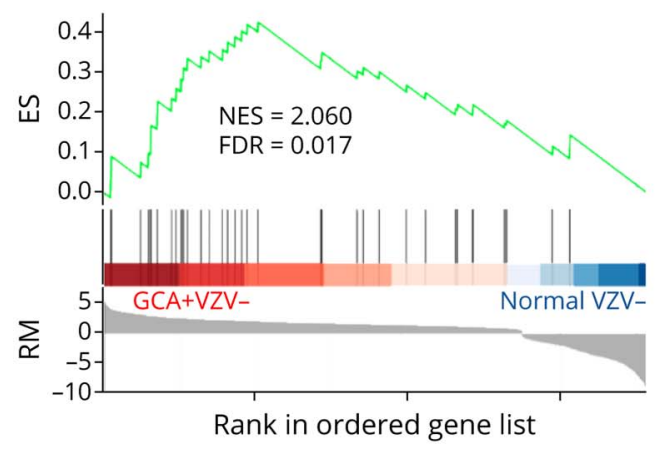

B
Fcy receptor-mediated phagocytosis in macrophages and neutrophilsFcy receptor-mediated phagocytosis in macrophages and monocytes-
Production of nitric oxide and reactive oxygen species in macrophagesNeuroinflammation signaling pathwayT cell exhaustion signaling pathway-

PD-1, PD-L1 cancer immunotherapy pathwayRole of NFAT in regulation of immune responseLeukocyte extravasation signaling-
Th1 pathwayTh1 pathway-
CD28 signaling in Thelper cells
Th2 pathway-
Antigen presentation pathway-
IL-8 signaling

C.b Toll-like receptor signaling

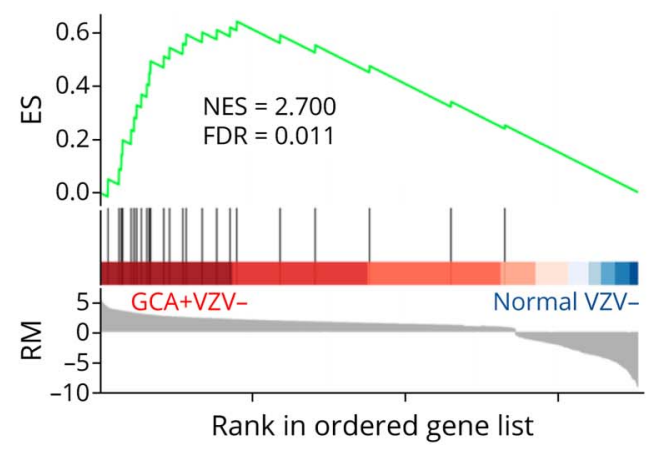

(A) Scatterplot depicting significantly upregulated (red) and downregulated (blue) genes in GCA/VZV-negative TAs compared with control/VZV-negative TAs. (B) Top canonical pathways identified in GCA/VZV-negative TAs vs control/VZV-negative TAs showed signatures of viral infections and immune responses. (C) In GCA/VZV-negative TAs, gene sets were positively enriched for defense response to virus, viral life cycle, and toll-like receptor signaling. The y-axis represents ES and RM (significant genes ranked by fold-change); $x$-axis are genes (vertical black lines) represented in the ranked order gene sets. The green line connects points of ES and genes (NES). ES = enrichment score; FDR = false discovery rate; GCA = giant cell arteritis; IL = interleukin; NES = normalized enrichment score; NFAT = nuclear factor of activated T-cells; NF-KB = nuclear factor kappa B; RM = ranked metric; TA = temporal artery; VZV, varicella zoster virus.

with viral antigen found in systemic arteries, including TAs and the aorta. ${ }^{6-9}$ GCA is a systemic vasculitis of the elderly that produces a constellation of symptoms and signs including malaise, headache, TA tenderness, vision loss, elevated C-reactive protein, platelet count, and/or sedimentation rate that can progress to aortitis and stroke; TA biopsies show a transmural vasculitis with medial damage and giant/ epithelioid cells. Treatment is with corticosteroids to reduce the vascular inflammation; however, the exact cause of GCA is unknown. Previous studies demonstrating that VZV vasculopathy and GCA share clinicohistopathological features and that VZV antigen is found in a large number of GCA TAs suggest that a subset or all of GCA is a form of extracranial VZV vasculopathy. ${ }^{110}$ Several other studies did not find VZV antigen in TAs or found it at much lower frequencies ${ }^{11-15}$; these differences were attributed to nonspecific antigen staining, missed antigen detection because of skip lesions, differences in methodology, and/or insufficient sample sizes analyzed. In addition, it was speculated that if VZV antigen, as well as confirmatory VZV DNA, were present in the GCA TAs, it was merely a bystander and uninvolved in the pathogenesis. Thus, we examined the gene expression profiles of
GCA TAs to determine if human transcripts supportive of virus infection were present. Specifically, we completed whole human transcriptome and pathway analysis of GCA TAs with and without VZV antigen ( $\mathrm{n}=20$ in each group) and control TAs with and without VZV antigen $(\mathrm{n}=11$ and 20, respectively). Our results showed activation of viral and inflammatory pathways in GCA TAs, supporting a pathogenic role for a virus, most likely VZV, in this vasculitis.

The TempO-Seq targeted RNA sequencing assay used in our analysis provides a valuable tool to investigate human transcriptional pathways from archived FFPE tissues without the need for RNA extraction and conversion to complementary DNA; of note, this assay exclusively detects human transcripts, not viral transcripts. The results of TempO-seq analysis of FFPE tissue are comparable with that of fresh and frozen tissue ${ }^{16}$; using RNA samples, TempO-seq generates results, consistent with Affymetrix microarrays and Illumina whole transcriptome RNA-Seq. ${ }^{29}$ Furthermore, this assay has been used by other laboratories to identify biomarkers for aggressiveness of clear cell renal cell carcinoma and prognosis in FFPE tumor samples that were then verified by RNA-seq 
A

GCA/VZV-negative vs control/VZV-negative GCA/VZV-positive vs control/VZV-negative

B

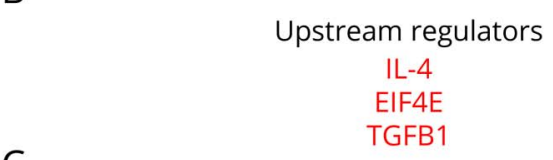

C
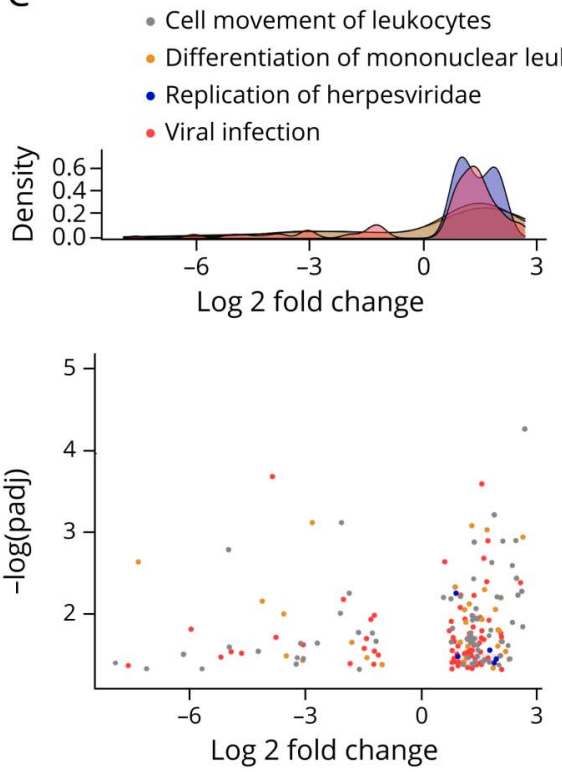

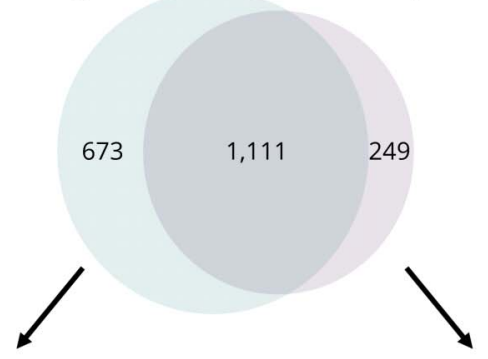

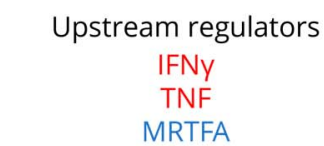
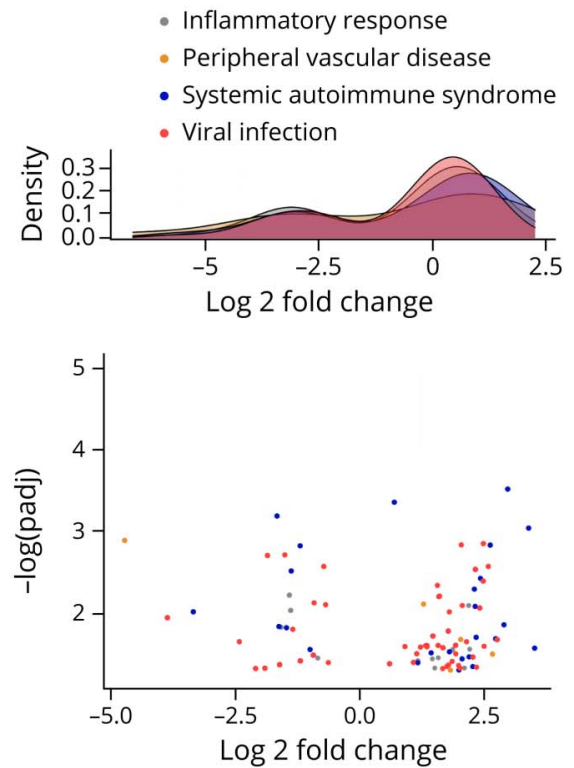

(A) In the analysis of GCA/VZV-negative TAs vs control/VZV-negative TAs compared with the analysis of GCA/ VZV-positive TAs vs control/VZV-negative TAs, a Venn diagram showed shared differentially expressed genes, as well as unique differentially expressed genes between the 2 analyses, suggesting that GCA TAs without or with VZV antigen may represent different stages of disease or different virus profiles, that may in part be determined by host responses. (B) Enrichment of unique GCA/VZV-negative genes identified IL4, EIF4E, and TGFB1 as key upstream regulators (left; red signifies activation). Upstream regulators for GCA VZV-positive unique genes were IFNy, TNF, and MRTFA (right; red signifies activation and blue signifies repression). (C) Volcano and density plot of enriched gene sets show distribution and direction of genes involved in cell movement of leukocytes, differentiation of mononuclear leukocytes, replication of Herpesviridae, and viral infection measured in GCA/VZV-negative unique genes (left). Volcano and density plot of enriched gene sets shows distribution and direction of genes involved in inflammatory response, peripheral vascular disease, systemic autoimmune syndrome, and viral infection measured in GCA/VZVpositive unique genes (right). GCA = giant cell arteritis; IFN = interferon; IL $=$ interleukin; $\mathrm{TA}=$ temporal artery; TNF = tumor necrosis factor; VZV varicella zoster virus. data from The Cancer Genome Atlas Kidney Renal Clear Cell Carcinoma. ${ }^{30}$ Without the limitation of access to fresh or frozen GCA TA tissues, we were able to sequence FFPE samples that have been archived up to 16 years. Importantly, slides can be immunostained for regions of pathology ( $\sim 10 \mathrm{~mm}^{2}, 5-7 \mu \mathrm{m}$ thickness) then scraped and analyzed. In this study, adjacent sections can be used in IHC to confirm transcriptional pathways, such as the IL-8 and neutrophil infiltration confirmation. Multiple findings of novel human gene expression pathways supporting a viral component in GCA and findings consistent with previous reports emerged from this study.

Our most salient finding was the detection of robust viral signatures in GCA/VZV-positive TAs compared with control/VZV-negative TAs, supporting previous IHC findings suggesting a viral etiology/contribution to GCA pathogenesis. $^{10,31}$ Specifically, we found human gene expression pathways associated with viral entry and replication and several shared genes in gene sets associated with viral infections, arterial disease, and immune cell activation, supporting a pathogenic role for virus in GCA TAs that contain VZV antigen. Like GCA/VZV-positive TAs, GCA/VZVnegative $\mathrm{TAs}$ showed transcriptional signatures associated with viral pathways and robust immune response pathways. These 2 groups were similar, sharing a majority of top canonical pathways. It is possible that individuals with GCA/ VZV-negative TAs have cleared viral antigen and now represent a cascade of pathology independent of active viral replication or another pathogen is involved; alternatively, VZV antigen on IHC may have been missed because of skip lesions. Indeed, a comparison of unique transcripts between control and GCA-positive TAs with or without VZV antigen revealed gene sets associated with generalized Herpesviridae infection, potentially representing a contribution of pathogens beyond VZV. Additional epidemiological studies support an association between $\mathrm{VZV}$, and to a lesser extent, herpes simplex virus (HSV), and GCA: (1) among 
16,686,345 US participants of whom 5,932 had GCA, complicated and uncomplicated herpes zoster was associated with a significantly increased risk of GCA (hazard ratios 1.99 and 1.42, respectively, in the Medicare cohort; 2.16 and 1.45, respectively, in the MarketScan cohort ${ }^{32}$; antiviral treatment was marginally associated with a decreased GCA risk in the Medicare cohort; and (2) among 3,026,005 British Columbia participants of which 4,315 had GCA, the prevalence of GCA in herpes zoster participants $(0.34 \%)$ was significantly higher than in the general population $(0.143 \%)^{33}$; the prevalence of GCA in HSV was also higher with the authors concluding that GCA seems to increase with herpetic infections, yet more significantly with zoster.

Aside from detecting novel viral and immune pathways, our results were consistent with previous GCA reports. Specifically, we uncovered pathways associated with Th1 activation, ${ }^{25}$ macrophage-mediated tissue disruption through reactive oxygen species, ${ }^{34}$ and neutrophil involvement. ${ }^{35,36}$ In addition, TLRs have been implicated in GCA pathogenesis ${ }^{26}$; Deng et al. ${ }^{25}$ found that TLR4 activation causes a transmural panarteritis through T-cell recruitment and activation. In this study, TLR4 is a predicted upstream regulator in all GCA TAs. We also found other predicted upstream regulators that have previously been associated with GCA risk or disease severity including INF $\gamma,{ }^{37,38} \mathrm{TNF}^{39,40} \mathrm{IL}-1 \beta{ }^{38,40} \mathrm{IL}-2,{ }^{37,38}$ and IL-6. ${ }^{40-42}$ Finally, multiple studies have shown an association with GCA susceptibility/severity and HLA-class II regions, namely, HLA-DRB1 (reviewed by Carmona et al.) ${ }^{27}$; we also measured higher levels of HLA-DRB1 transcripts in GCA/VZV-positive TAs compared with controls.

Previously, we found that $18 \%$ of TAs from normal participants contained VZV antigen with no associated GCA pathology (transmural inflammation, medial disruption, and giant and/or epithelioid cells). ${ }^{10}$ The significance of these observations was unclear: was the presence of VZV antigen incidental, not affecting the vascular environment, or because of nonspecific staining with the anti-VZV antibodies used? In our TempO-Seq assay, we found that compared with control/ VZV-negative TAs, control/VZV-positive TAs had pathways associated with viral infection, viral replication, budding of virus, and antigen presentation and crosstalk between dendritic cells and natural killer cells, supporting the ability of VZV to still elicit an antiviral response within arterial walls. These participants did not have clinical symptoms, signs, or GCA TA pathology. Yet, the presence of viral pathways raises the possibility that these participants may represent early GCA that may later progress to fulminant disease or that these individuals may have a greater ability to clear virus infection of arteries and/or more appropriately modulate the immune response, preventing aberrant inflammation leading to vasculitis. Importantly, the detection of viral-associated pathways in these TAs without the presence of robust inflammation suggests that the enriched pathways are unlikely nonspecific signatures of infiltrating immune cells and argues against nonspecific VZV antigen staining.
Overall, gene expression and pathway analysis indicated strong evidence for a viral contribution to the pathogenesis of biopsyconfirmed GCA. Although this assay did not specifically identify the virus, among all published viral causes of GCA, VZV is the most biologically plausible, given the compelling clinicohistopathological overlap between VZV vasculopathy and GCA. The unbiased finding of viral contributions and host antiviral responses support the immunohistochemical observations of the presence of VZV antigen in GCA TAs (confirmed in many cases by the presence of VZV DNA) and, more specifically, support the ability of virus to induce vascular inflammation that is characteristic of GCA pathogenesis. These findings have the potential to change the standard of care for patients with GCA. Currently, patients with GCA are treated with long-term corticosteroids to reduce the vascular inflammation but not the underlying cause of inflammation; however, steroids have significant side effects particularly in the vulnerable elderly population, such as potentiation of virus infection, accelerated osteoporosis, hypertension, hyperglycemia, and irritability. Furthermore, approximately $50 \%$ of patients on corticosteroids continue to worsen clinically (vision loss, stroke) or have recurrent disease when steroids are tapered. ${ }^{43}$ The addition of an antiviral agent that would treat the underlying cause of vascular inflammation may improve patient outcomes, thus warranting multicenter clinical trials to determine antiviral drug dose and duration, as well as concomitant administration with corticosteroids.

\section{Acknowledgment}

The authors would like to thank Cathy Allen for manuscript preparation and the BioFrontiers Institute Next-Gen Sequencing Core Facility which performed the Illumina sequencing. Coauthor Dr. Cohrs passed away prior to manuscript publication.

\section{Study Funding}

This work was supported by Grant AG032958 from the NIH to M.A. Nagel, R.J. Cohrs, and R. Mahalingam.

\section{Disclosure}

The authors report no disclosures. Go to Neurology.org/NN for full disclosures.

\section{Publication History}

Received by Neurology: Neuroimmunology \& Neuroinflammation April 20, 2021. Accepted in final form June 28, 2021.

\section{Appendix Authors}

\begin{tabular}{lll}
\hline Name & Location & Contribution \\
\hline $\begin{array}{l}\text { Andrew N. } \\
\text { Bubak, PhD }\end{array}$ & $\begin{array}{l}\text { University of } \\
\text { Colorado, } \\
\text { Aurora }\end{array}$ & $\begin{array}{l}\text { Designed and conceptualized the } \\
\text { study, performed experiments, } \\
\text { analyzed the data, discussed and } \\
\text { interpreted the findings, and drafted } \\
\text { and revised the article for intellectual } \\
\end{array}$ \\
& content \\
\hline
\end{tabular}

\begin{tabular}{lll}
\hline $\begin{array}{l}\text { Teresa } \\
\text { Mescher, BS }\end{array}$ & $\begin{array}{l}\text { University of } \\
\text { Colorado, } \\
\text { Aurora }\end{array}$ & $\begin{array}{l}\text { Performed experiments and revised } \\
\text { the article for intellectual content }\end{array}$ \\
& &
\end{tabular}


Appendix (continued)

\begin{tabular}{|c|c|c|}
\hline Name & Location & Contribution \\
\hline $\begin{array}{l}\text { Michael } \\
\text { Mariani, PhD }\end{array}$ & $\begin{array}{l}\text { University of } \\
\text { Vermont, } \\
\text { Burlington }\end{array}$ & $\begin{array}{l}\text { Performed bioinformatics analyses } \\
\text { and revised the article for intellectual } \\
\text { content }\end{array}$ \\
\hline $\begin{array}{l}\text { Seth E. Frietze, } \\
\text { PhD }\end{array}$ & $\begin{array}{l}\text { University of } \\
\text { Vermont, } \\
\text { Burlington }\end{array}$ & $\begin{array}{l}\text { Analyzed the data and interpreted the } \\
\text { findings and revised the article for } \\
\text { intellectual content }\end{array}$ \\
\hline $\begin{array}{l}\text { James E. } \\
\text { Hassell, Jr., PhD }\end{array}$ & $\begin{array}{l}\text { University of } \\
\text { Colorado, } \\
\text { Aurora }\end{array}$ & $\begin{array}{l}\text { Performed bioinformatics analyses } \\
\text { and revised the article for intellectual } \\
\text { content }\end{array}$ \\
\hline $\begin{array}{l}\text { Christy S. } \\
\text { Niemeyer, PhD }\end{array}$ & $\begin{array}{l}\text { University of } \\
\text { Colorado, } \\
\text { Aurora }\end{array}$ & $\begin{array}{l}\text { Performed experiments and revised } \\
\text { the article for intellectual content }\end{array}$ \\
\hline $\begin{array}{l}\text { Christina N. } \\
\text { Como, BA }\end{array}$ & $\begin{array}{l}\text { University of } \\
\text { Colorado, } \\
\text { Aurora }\end{array}$ & $\begin{array}{l}\text { Performed experiments and revised } \\
\text { the article for intellectual content }\end{array}$ \\
\hline $\begin{array}{l}\text { Anna M. } \\
\text { Burnet, BS }\end{array}$ & $\begin{array}{l}\text { University of } \\
\text { Colorado, } \\
\text { Aurora }\end{array}$ & $\begin{array}{l}\text { Performed experiments and revised } \\
\text { the article for intellectual content }\end{array}$ \\
\hline $\begin{array}{l}\text { Prem S. } \\
\text { Subramanian, } \\
\text { MD, PhD }\end{array}$ & $\begin{array}{l}\text { University of } \\
\text { Colorado, } \\
\text { Aurora }\end{array}$ & $\begin{array}{l}\text { Analyzed the data and interpreted the } \\
\text { findings and revised the article for } \\
\text { intellectual content }\end{array}$ \\
\hline $\begin{array}{l}\text { Randall J. } \\
\text { Cohrs, PhD }\end{array}$ & $\begin{array}{l}\text { University of } \\
\text { Colorado, } \\
\text { Aurora }\end{array}$ & $\begin{array}{l}\text { Analyzed the data and interpreted the } \\
\text { findings and revised the article for } \\
\text { intellectual content }\end{array}$ \\
\hline $\begin{array}{l}\text { Ravi } \\
\text { Mahalingam, } \\
\text { PhD }\end{array}$ & $\begin{array}{l}\text { University of } \\
\text { Colorado, } \\
\text { Aurora }\end{array}$ & $\begin{array}{l}\text { Analyzed the data and interpreted the } \\
\text { findings and revised the article for } \\
\text { intellectual content }\end{array}$ \\
\hline $\begin{array}{l}\text { Maria A. Nagel, } \\
\text { MD }\end{array}$ & $\begin{array}{l}\text { University of } \\
\text { Colorado, } \\
\text { Aurora }\end{array}$ & $\begin{array}{l}\text { Designed and supervised the study, } \\
\text { analyzed the data, discussed and } \\
\text { interpreted the findings, and revised } \\
\text { the article for intellectual content }\end{array}$ \\
\hline
\end{tabular}

\section{References}

1. Gilden D, White T, Khmeleva N, et al. Prevalence and distribution of VZV in temporal arteries of patients with giant cell arteritis. Neurology. 2015;84(19):1948-1955.

2. Al-Abdulla NA, Rismondo V, Minkowski JS, Miller NR. Herpes zoster vasculitis presenting as giant cell arteritis with bilateral internuclear ophthalmoplegia. Am J Ophthalmol. 2002;134(6):912-914.

3. Al-Abdulla NA, Kelley JS, Green WR, Miller NR. Herpes zoster vasculitis presenting as giant cell arteritis with choroidal infarction. Retina. 2003;23(4):567-569.

4. Nagel MA, Khmeleva N, Boyer PJ, Choe A, Bert R, Gilden D. Varicella zoster virus in the temporal artery of a patient with giant cell arteritis. J Neurol Sci. 2013;335(1-2):228-230.

5. Nagel MA, Bennett JL, Khmeleva N, et al. Multifocal VZV vasculopathy with temporal artery infection mimics giant cell arteritis. Neurology. 2013;80(22):2017-2021.

6. Victor DI, Green WR. Temporal artery biopsy in herpes zoster ophthalmicus with delayed arteritis. Am J Ophthalmol. 1976;82(4):628-630.

7. Nagel MA, Traktinskiy I, Stenmark KR, Frid MG, Choe A, Gilden D. Varicella-zoster virus vasculopathy: immune characteristics of virus-infected arteries. Neurology. 2013;80(1):62-68.

8. Nagel MA, Lenggenhager $\mathrm{D}$, White $\mathrm{T}$, et al. Disseminated VZV infection and asymptomatic VZV vasculopathy after steroid abuse. J Clin Virol. 2015;66:72-75.

9. Gilden D, White T, Boyer PJ, et al. Varicella zoster virus infection in granulomatous arteritis of the aorta. J Infect Dis. 2016;213(12):1866-1871.

10. Gilden D, White T, Khmeleva N, Boyer PJ, Nagel MA. VZV in biopsy-positive and -negative giant cell arteritis: analysis of 100+ temporal arteries. Neurol Neuroimmunol Neuroinflamm. 2016;3(2);e216. doi: 10.1212/NXI.0000000000000216

11. Muratore F, Croci S, Tamagnini I, et al. No detection of varicella-zoster virus in temporal arteries of patients with giant cell arteritis. Semin Arthritis Rheum. 2017;47(2):235-240.

12. Buckingham EM, Foley MA, Grose C, et al. Identification of herpes zoster-associated temporal arteritis among cases of giant cell arteritis. Am J Ophthalmol. 2018;187:51-60.

13. Solomon IH, Docken WP, Padera RF Jr. Investigating the association of giant cell arteritis with varicella zoster virus in temporal artery biopsies or ascending aortic resections. J Rheumatol. 2019;46(12):1614-1618.
14. Sammel AM, Smith S, Nguyen K, et al. Assessment for varicella zoster virus in patients newly suspected of having giant cell arteritis. Rheumatology (Oxford). 2020;59(8): 1992-1996.

15. Verdijk RM, Ouwendijk WJD, Kruipers RWAM, Verjans GMGM. No evidence of varicella-zoster virus infection in temporal artery biopsies of anterior ischemic optic neuropathy patients with and without giant cell arteritis. J Infect Dis. 2021;223(1): 109-112.

16. Trejo CL, Babić M, Imler E, et al. Extraction-free whole transcriptome gene expression analysis of FFPE sections and histology-directed subareas of tissue. PLoS One. 2019;14(2):e0212031.

17. Langmead B, Trapnell C, Pop M, Salzberg SL. Ultrafast and memory-efficient alignment of short DNA sequences to the human genome. Genome Biol. 2009;10(3): R25.

18. Varet H, Brillet-Guéguen L, Coppée JY, Dillies MA. SARTools: a DESeq2-and EdgeRbased R pipeline for comprehensive differential analysis of RNA-Seq data. PLoS One. 2016;11(6):e0157022.

19. Robinson MD, McCarthy DJ, Smyth GK. edgeR: a Bioconductor package for differential expression analysis of digital gene expression data. Bioinformatics. 2010; 26(1):139-140.

20. Love MI, Huber W, Anders S. Moderated estimation of fold change and dispersion for RNA-Seq data with DESeq2. Genome Biol. 2014;15(12):550.

21. Yu G, Wang L, Han Y, He Q. clusterProfiler: an R package for comparing biological themes among gene clusters. OMICS. 2012;16(5):284-287.

22. Yu G, Wang LG, Yan GR, He QY. DOSE: an R/Bioconductor package for disease ontology semantic and enrichment analysis. Bioinformatics. 2015;31(4):608-609.

23. R Core Team. $R$ : A Language and Environment for Statistical Computing. R Foundation for Statistical Computing; 2020. Available at: R-project.org/.

24. Brass AL, Huang I-C, Benita Y, et al. The IFITM proteins mediate cellular resistance to influenza A H1N1 virus, West Nile virus, and dengue virus. Cell. 2009;139(7): 1243-1254.

25. Deng J, Ma-Krupa W, Gewirtz AT, Younge BR, Goronzy JJ, Weyand CM. Toll-like receptors 4 and 5 induce distinct types of vasculitis. Circ Res. 2009;104(4):488-495.

26. O'Neill L, Molloy ES. The role of toll like receptors in giant cell arteritis. Rheumatology (Oxford). 2016;55(11):1921-1931.

27. Carmona FD, Gonzalez-Gay MA, Martín J. Genetic component of giant cell arteritis. Rheumatology (Oxford). 2014;53(1):6-18.

28. Minami T, Kuwahara K, Nakagawa Y, et al. Reciprocal expression of MRTF-A and myocardin is crucial for pathological vascular remodelling in mice. EMBO J. 2012 ; 31(23):4428-4440

29. Bushel PR, Paules RS, Auerbach SS. A comparison of the TempO-Seq S1500+ platform to RNA-Seq and microarray using rat liver mode of action samples. Front Genet. 2018;9:485.

30. Batai K, Imler E, Pangilinan J, et al. Whole-transcriptome sequencing identified gene expression signatures associated with aggressive clear cell renal cell carcinoma. Genes Cancer. 2018;9(5-6):247-256.

31. Blackmon AM, Como CN, Bubak AN, Mescher T, Jones D, Nagel MA. Varicella zoster virus alters expression of cell adhesion proteins in human perineurial cells via interleukin 6. J Infect Dis. 2019;220(9):1453-1461.

32. England BR, Mikuls TR, Xie F, Yang S, Chen L, Curtis JR. Herpes zoster as a risk factor for incident giant cell arteritis. Arthritis Rheum. 2017;69(12):2351-2358.

33. Lee DH, Iovieno A, Sheldon CA. Is there an association between herpetic infections and giant cell arteritis? A population-based study. J Clin Med. 2021;10(1):63.

34. Rittner HL, Kaiser M, Brack A, Szweda LI, Goronzy JJ, Weyand CM. Tissuedestructive macrophages in giant cell arteritis. Circ Res. 1999;84(9):1050-1080.

35. Nadkarni S, Dalli J, Hollywood J, Mason JC, Dasgupta B, Perretti M. Investigational analysis reveals a potential role for neutrophils in giant-cell arteritis disease progression. Circ Res. 2014;114(2):242-248.

36. Wang L, Ai Z, Khoyratty T, et al. ROS-producing immature neutrophils in giant cell arteritis are linked to vascular pathologies. CI Insight. 2020;5(20):e139163.

37. Weyand CM, Hicok KC, Hunder GG, Goronzy JJ. Tissue cytokine patterns in patients with polymyalgia rheumatic and giant cell arteritis. Ann Intern Med. 1994; 121(7):484-491.

38. Weyand CM, Tetzlaff N, Björnsson J, Brack A, Younge B, Goronzy JJ. Disease patterns and tissue cytokine profiles in giant cell arteritis. Arthritis Rheum. 1997;40(1) $19-26$.

39. Mattey DL, Hajeer AH, Dababneh A, et al. Association of giant cell arteritis and polymyalgia rheumatica with different tumor necrosis factor microsatellite polymorphisms. Arthritis Rheum. 2000;43(8):1749-1755.

40. Hernandez-Rodriguez J, Segarra M, Vilardell C, et al. Tissue production of proinflammatory cytokines (IL-1 $\beta, T N F \alpha$ and IL-6) correlates with the intensity of the systemic inflammatory response and with corticosteroid requirements in giant-cell arteritis. Rheumatology (Oxford). 2004;43(3):294-301.

41. Dasgupta B, Panayi GS. Interleukin-6 in serum of patients with polymyalgia rheumatica and giant cell arteritis. Br J Rheumatol. 1990;29(6):456-458.

42. Roche NE, Fulbright JW, Wagner AD, Hunder GG, Goronzy JJ, Weyand CM. Correlation of interleukin- 6 production and disease activity in polymyalgia rheumatica and giant cell arteritis. Arthritis Rheum. 1993;36(9):1286-1294.

43. Fraser JA, Weyand CM, Newman NJ, Biousse V. The treatment of giant cell arteritis. Rev Neurol Dis. 2008;5(3):140-152. 


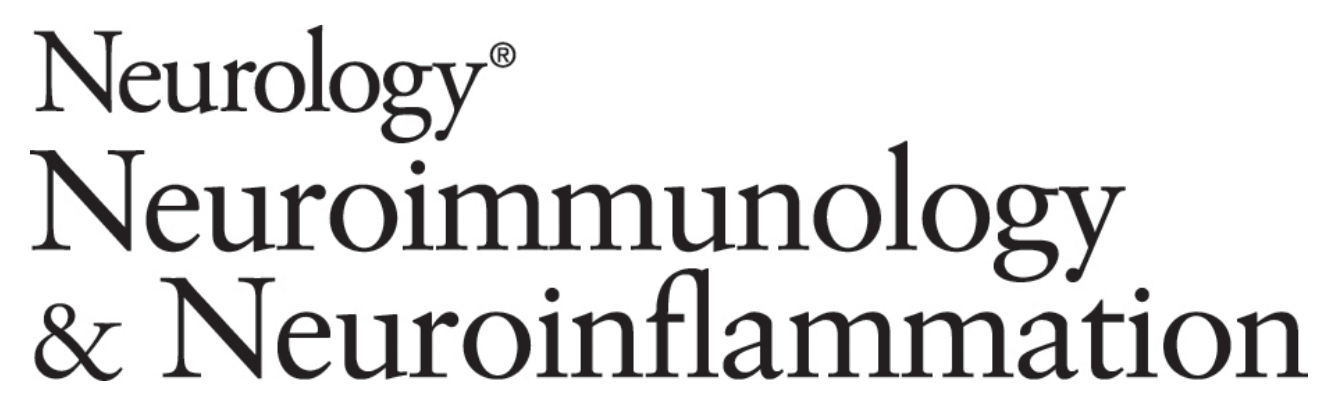

Targeted RNA Sequencing of Formalin-Fixed, Paraffin-Embedded Temporal Arteries From Giant Cell Arteritis Cases Reveals Viral Signatures Andrew N. Bubak, Teresa Mescher, Michael Mariani, et al. Neurol Neuroimmunol Neuroinflamm 2021;8; DOI 10.1212/NXI.0000000000001078

This information is current as of September 7, 2021

Neurol Neuroimmunol Neuroinflamm is an official journal of the American Academy of Neurology. Published since April 2014, it is an open-access, online-only, continuous publication journal. Copyright Copyright $\odot 2021$ The Author(s). Published by Wolters Kluwer Health, Inc. on behalf of the American Academy of Neurology.. All rights reserved. Online ISSN: 2332-7812.

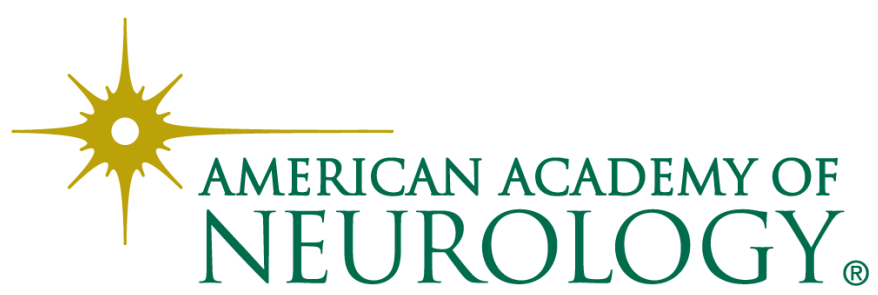




\section{Updated Information \& Services}

References

Subspecialty Collections

Permissions \& Licensing

Reprints including high resolution figures, can be found at: http://nn.neurology.org/content/8/6/e1078.full.html

This article cites 42 articles, 6 of which you can access for free at: http://nn.neurology.org/content/8/6/e1078.full.html\#\#ref-list-1

This article, along with others on similar topics, appears in the following collection(s):

\section{All Cerebrovascular disease/Stroke}

http://nn.neurology.org//cgi/collection/all_cerebrovascular_disease_str oke

All Immunology

http://nn.neurology.org//cgi/collection/all_immunology

Gene expression studies

http://nn.neurology.org//cgi/collection/gene_expression_studies

Vasculitis

http://nn.neurology.org//cgi/collection/vasculitis

Viral infections

http://nn.neurology.org//cgi/collection/viral_infections

Information about reproducing this article in parts (figures,tables) or in its entirety can be found online at:

http://nn.neurology.org/misc/about.xhtml\#permissions

Information about ordering reprints can be found online:

http://nn.neurology.org/misc/addir.xhtml\#reprintsus

Neurol Neuroimmunol Neuroinflamm is an official journal of the American Academy of Neurology.

Published since April 2014, it is an open-access, online-only, continuous publication journal. Copyright

Copyright $\odot 2021$ The Author(s). Published by Wolters Kluwer Health, Inc. on behalf of the American

Academy of Neurology.. All rights reserved. Online ISSN: 2332-7812.

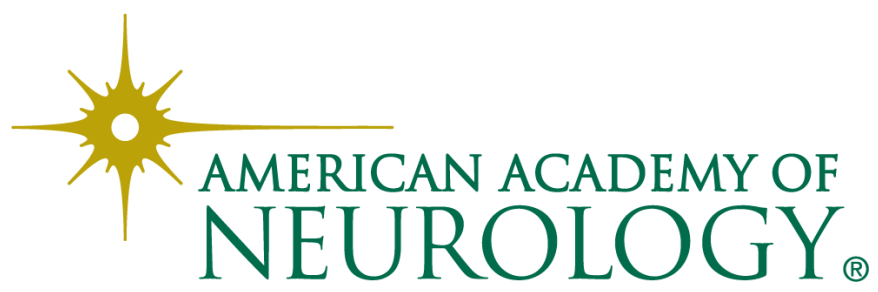

